BNL-114389-2017-JA

\title{
Observational study of the relationship between entrainment rate and relative dispersion in deep convective clouds
}

X. Guo, Y. Liu

To be published in "Atmospheric Research"

September 2017

Environmental \& Climate Sciences Department

Brookhaven National Laboratory

\author{
U.S. Department of Energy \\ USDOE Office of Science (SC), \\ Biological and Environmental Research (BER) (SC-23)
}

Notice: This manuscript has been authored by employees of Brookhaven Science Associates, LLC under Contract No. DE-SC0012704 with the U.S. Department of Energy. The publisher by accepting the manuscript for publication acknowledges that the United States Government retains a non-exclusive, paid-up, irrevocable, world-wide license to publish or reproduce the published form of this manuscript, or allow others to do so, for United States Government purposes. 


\section{DISCLAIMER}

This report was prepared as an account of work sponsored by an agency of the United States Government. Neither the United States Government nor any agency thereof, nor any of their employees, nor any of their contractors, subcontractors, or their employees, makes any warranty, express or implied, or assumes any legal liability or responsibility for the accuracy, completeness, or any third party's use or the results of such use of any information, apparatus, product, or process disclosed, or represents that its use would not infringe privately owned rights. Reference herein to any specific commercial product, process, or service by trade name, trademark, manufacturer, or otherwise, does not necessarily constitute or imply its endorsement, recommendation, or favoring by the United States Government or any agency thereof or its contractors or subcontractors. The views and opinions of authors expressed herein do not necessarily state or reflect those of the United States Government or any agency thereof. 


\section{Observational study of the relationship between entrainment rate and relative dispersion in deep convective clouds}

Xiaohao Guo ${ }^{1,2}$, Chunsong Lu ${ }^{1,3}$, Tianliang Zhao ${ }^{1}$, Yangang Liu ${ }^{4}$, Guang Jun Zhang ${ }^{5}$, and Shi Luo ${ }^{1}$

${ }^{1}$ Key Laboratory of Meteorological Disaster, Ministry of Education (ELME)/ Joint International Research Laboratory of Climate and Environment Change (ILCEC)/ Collaborative Innovation Center on Forecast and Education of Meteorological Disasters (CIC-FEMD)/ Key Laboratory for Aerosol-Cloud-Precipitation of China Meteorological Administration, Nanjing University of Information Science and Technology, Nanjing, China, ${ }^{2}$ Suzhou Meteorological Bureau, Suzhou, China, ${ }^{3}$ Ministry of Education Key Laboratory for Earth System Modeling, and Department for Earth System Science, Tsinghua University, Beijing, China, ${ }^{4}$ Environmental and Climate Science Department, Brookhaven National Laboratory, Upton, USA, ${ }^{5}$ Scripps Institution of Oceanography, University of California, San Diego, USA

Corresponding to: C. S. Lu, luchunsong110@gmail.com; T. L. Zhao, tlzhao@ nuist.edu.cn 
Abstract: This study investigates the influence of entrainment rate $(\lambda)$ on relative dispersion $(\varepsilon)$ of cloud droplet size distributions (CDSD) in the 99 growing precipitating deep convective clouds during TOGA-COARE. The results show that entrainment suppresses $\varepsilon$, which is opposite to the traditional understanding that entrainment-mixing broadens CDSD. To examine how the relationship between $\varepsilon$ and $\lambda$ is affected by droplets with different sizes, CDSDs are divided into three portions with droplet radius $<3.75 \mu \mathrm{m}\left(N_{1}\right)$, radius in the range of 3.75-12.75 $\mu \mathrm{m}\left(N_{2}\right)$ and $12.75-23.25 \mu \mathrm{m}\left(N_{3}\right)$, respectively. The results indicate that although the droplet concentration at different sizes generally decrease simultaneously as $\lambda$ increases, the variation of standard deviation $(\sigma)$ depends mainly on $N_{3}$, while the mean radius $\left(r_{\mathrm{m}}\right)$ decreases with decreasing $N_{3}$, but increases with decreasing $N_{1}$. So the influence of entrainment on CDSD causes a more dramatical decrease in $\sigma$ than that in $r_{\mathrm{m}}$, and further leads to the decrease of $\varepsilon$ as entrainment enhances. In addition, a conceptual model of CDSD evolution during entrainment-mixing processes is developed to illustrate the possible scenarios entailing different relationships between $\varepsilon$ and $\lambda$. The number concentration of small droplets and the degree of evaporation of small droplets are found to be key factors that shift the sign (i.e., positive or negative) of the $\varepsilon-\lambda$ relationship. 


\section{Introduction}

Clouds play significant roles in radiative transfer and the earth's radiative energy balance [Lin et al., 2015; Shi and Liu, 2016; Zhang et al., 2017a]. The cloud radiative properties are closely related to cloud droplet size distributions (CDSDs) and the corresponding microphysical properties [Zhang et al., 2016, 2017b; Chen et al., 2017]. Relative dispersion $(\varepsilon)$ of CDSD, the ratio of standard deviation $(\sigma)$ to mean radius $\left(r_{\mathrm{m}}\right)$, is a relative measure of spectral width [Liu et al., 2014] and an important microphysical parameter affecting cloud radiative properties [Martin et al., 1994; Liu and Daum, 2000, 2002; Peng and Lohmann, 2003]. Liu et al., [2008] showed that using an unrealistic $\varepsilon$ in climate models, such as assuming a monodisperse cloud, can lead to significant overestimation of cloud albedo and further result in a negative bias of global mean estimates of shortwave cloud radiative forcing. In addition, $\varepsilon$ is closely linked to the development of precipitation, and thus is essential to accurate parameterization of precipitation formation [Seifert and Beheng, 2001]. Liu and Daum [2005] introduced $\varepsilon$ into the parameterization of autoconversion process and found that autoconversion rate increases with increasing $\varepsilon$. Xie et al., [2013] further applied different parameterizations of $\varepsilon$ into the Weather Research and Forecast (WRF) model, and found that surface precipitation was sensitive to $\varepsilon$ parameterizations.

Due to the importance of $\varepsilon$, much effort has been devoted to studying the factors affecting $\varepsilon$, such as aerosol loading, vertical velocity and entrainment. For example, some scientists found a positive correlation between $\varepsilon$ and aerosol loading [e.g., McFarquhar and Heymsfield, 2001; Liu and Daum, 2002; Wood et al., 2002; Yum and Hudson, 2005], whereas others found a negative correlation [e.g., Ma et al., 2010; Lu et al., 2012a] or no correlation between them [e.g., Zhao et al., 2006; Tas et al., 2015]. Another factor affecting the correlation between $\varepsilon$ and aerosol loading is vertical velocity, which was theoretically [Liu et al., 2006] and observationally [Lu et al., 2012a] shown to reduce $\varepsilon$. Recently, Chen et al., [2016] suggested that the relationship of $\varepsilon$ to aerosol concentration and vertical velocity is strongly regime-dependent: given an updraft velocity, $\varepsilon$ first increases with increasing aerosol loading in the aerosol-limited regime, peaks in the transitional regime and then decreases with further increase in aerosol loading in the updraftlimited regime.

In addition to aerosols and vertical velocity, entrainment of dry air into clouds is another important factor known to affect CDSD [Lu et al., 2013; Kumar et al., 2014; Yang et al., 2016; 
Chen et al., 2017]. Microphysical properties of CDSDs in real-world atmospheric clouds are quite different from those of adiabatic CDSD [Grabowski and Pawlowska, 1993; Liu and Hallet, 1998; Yum et al., 2015], and are closely related to turbulent entrainment-mixing [Su et al., 1998; Lasher-Trapp et al., 2005; Lu et al., 2013; Tölle and Krueger, 2014; Beals et al., 2015; Xu and Xue, 2015]. Based on numerical simulations, Su et al., [1998] and Burnet and Brenguier [2007] revealed that, without entrainment-mixing process, simulated CDSDs were all narrower than in realistic clouds. Burnet and Brenguier [2007] suggested that entrainment-mixing processes produced plenty of droplet trajectory and water vapor supersaturation scenarios, hence leading to broad and multimodal CDSD and large $\varepsilon$. The observations of Lu et al., [2013] showed that $\varepsilon$ increased in the process of entrainment-mixing, and $\sigma$ remained almost constant between 0.75 and $1 \mu \mathrm{m}$. The roughlyconstant $\sigma$ was similar to that found by Pawlowska et al., [2006]. Therefore, Lu et al., [2013] attributed the increase of $\varepsilon$ in the entrainment-mixing processes to the decrease of $r_{\mathrm{m}}$. Prabha et al., [2012] studied the relationships of $\varepsilon$ to big and small droplets as influenced by entrainment-mixing processes represented by adiabatic fraction, and found that the relationships were complicated and different between premonsoon and monsoon clouds. Pinsky et al., [2016] analyzed the evolution of $\varepsilon$ in the homogeneous entrainment-mixing processes and found that $\varepsilon$ increased at first and then approached a constant value.

Despite these efforts devoted to this topic, our understanding of the effect of entrainment on $\varepsilon$ is still far from complete, especially for deep convective clouds. Here we examine the relationship between $\varepsilon$ and entrainment rate $(\lambda)$ in the deep convection observed by aircraft during the TOGA-COARE (Tropical Ocean Global Atmosphere Coupled Ocean Atmosphere Response Experiment, 1992-1993) field campaign. For convective clouds, $\lambda$ quantitatively represents the ratio of the entrained dry air mass (flux) to the cloud mass (flux) per unit height and is an important parameter in convection parameterizations [Del Genio and $\mathrm{Wu}, 2010$; Zhang et al., 2015]. Tahe authors knowledgethis is the first observationstudy orthe relationshipbetween $\varepsilon$ and $\lambda$ in deep convective clouds. The rest of the paper is organized as follows. Section 2 briefly describes the TOGA-COARE campaign and the method for estimating $\lambda$. Section 3 presents the main results, including the influence of $\lambda$ on $\varepsilon$ and the corresponding mechanisms. Conclusions are presented in Section 4. 


\section{Data and Approach}

\subsection{TOGA-COARE}

During TOGA-COARE [Webster and Lucas, 1992], Electra research aircraft from NCAR (National Center for Atmospheric Research) carried out 32 observation missions to probe the physical parameters $(1 \mathrm{~Hz})$ in deep convective clouds. CDSDs were detected by the Forward Scattering Spectrometer Model-100 probe with 15 bins to calculate cloud microphysical parameters including liquid water content (LWC), number concentration $(N), \varepsilon, \sigma$ and $r_{\mathrm{m}}$. Some other information about measurement ranges and errors of instruments used in this study can be found in Guo et al., [2015]. The following criteria are used to identify individual clouds. CDSDs with LWC larger than $0.001 \mathrm{~g} \mathrm{~m}^{-3}$ and $N$ of cloud droplets greater than $10 \mathrm{~cm}^{-3}$ are considered to be cloud records [Lu et al., 2012b; Lu et al., 2013]. The percent of updraft in an individual cloud must be larger than $80 \%$ to be selected as growing clouds [Gerber et al., 2008]. Cloud penetration time must exceed 5 s to obtain large clouds [Norgren et al., 2016]. There were a total of 99 precipitating clouds at the $\sim 3000 \mathrm{~m}$ observation height satisfying these criteria and the following analyses are based on these clouds. The mean value, maximum value and standard deviation of the vertical velocities in the 99 clouds are $1.1 \mathrm{~m} \mathrm{~s}^{-1}, 6.0 \mathrm{~m} \mathrm{~s}^{-1}$ and $1.1 \mathrm{~m} \mathrm{~s}^{-1}$, respectively. The number of clouds herein is slightly larger than that (92) in Guo et al., [2015] at the $~ 3000 \mathrm{~m}$ height under the similar criteria, because the clouds observed in the height range of 2900-3100 m are analyzed in this study, while Guo et al., [2015] only analyzed the clouds in the range of 2950-3050 m. In addition, the mean in-cloud buoyancy is larger than $0 \mathrm{~m} \mathrm{~s}^{-2}$ herein. The purpose is to select more relatively appropriate growing cloud samples.

\subsection{Approach for estimating $\lambda$}

Guo et al., [2015] estimated $\lambda$ in growing clouds during TOCA-COARE using the traditional bulk-plume method [Gerber et al., 2008]:

$$
\lambda=\frac{\partial \phi t}{\partial z} \frac{1}{\phi_{t}-\phi t},
$$

where $z$ denotes height, $\phi_{\mathrm{c}}$ and $\phi_{\mathrm{e}}$ represent conserved physical quantities in cloud and environment, respectively. In our study, moist static energy (MSE) is used as the conserved 
quantity when studying entrainment-mixing processes based on many previous studies [e.g., Khairoutdinov and Randall, 2006; Luo et al., 2010; Martin and Paul, 2013; Böing et al., 2014].

In Equation (1), $\partial \phi_{c} / \partial z$ is the vertical gradient of $\phi_{c}$ in cloud. Assuming $\lambda$ is homogeneously distributed from cloud base to observational level, i.e., the obtained $\lambda$ value is invariant within this height range, the average MSE on observational height and cloud base is used for $\phi_{c}$. The environmental $\phi_{e}$ is taken as the average MSE within the same height range from the vertical profile of environmental MSE that is far away from the cloud edges. See details of the calculations in Guo et al., [2015]. The estimated $\lambda$ values are used to study the influence of entrainment rate on $\varepsilon$. When $\lambda$ is estimated assuming entrained air is near cloud edge, the results are similar to Section 3 hereinafter, and therefore are not included in this study.

\section{Results}

\subsection{Influence of entrainment on $\varepsilon$}

Entrainment can significantly influence the microphysical characteristics of CDSD. Figure 1 shows the relationship of $\varepsilon$ to $\lambda$ for the total 99 clouds. The correlation is negative with a correlation coefficient $(R)$ of -0.37 , suggesting that stronger entrainment reduces. This result is opposite to the traditional understanding that entrainment-mixing broadens CDSD [Su et al., 1998; Lasher-Trapp et al., 2005; Lu et al., 2013; Tölle and Krueger, 2014]. It is generally believed that CDSD broadens toward small cloud droplets and thus $\varepsilon$ increases in thentrainment-mixing processes. For example, Lu et al., [2013] analyzed the correlation between $\varepsilon$ and $\lambda$ in shallow cumulus and they found that $\varepsilon$ increases with increasing $\lambda$. But this study shows the inhibition effect of entrainment on $\varepsilon$. As mentioned in the introduction, $\varepsilon$ is crucial for precipitation. So the influence of entrainment on $\varepsilon$ is also very important. The mechanisms of how entrainment affects CDSD and $\varepsilon$ are needed to be examined.

\subsection{Influence of entrainment on CDSD}

The relationship between $\varepsilon$ and $\lambda$ depends on the influence of entrainment on CDSD. Figure 2 shows the influence of $\lambda$ on CDSD. Figure 2a is plotted by averaging CDSD for three $\lambda$ bins with an identical bin width in the range of $\sim 0.10 \mathrm{~km}^{-1}$ to $\sim 0.40 \mathrm{~km}^{-1}$. The numbers of clouds in these

three bins are 10,50 and 39, respectively. With the increase off the whole droplet size 
distributions move downward. Figure $2 b$ shows the CDSDs averaged for twelve $\lambda$ bins with an identical bin width. The numbers of clouds in these twelve bins are respectively, 3, 1, 5, 7, 14, 10, $12,11,13,6,12$ and 5. The first two bins are combined into one bin because there are too few samples in the individual bins. The variations of CDSDs are quite complicated in Figure $2 \mathrm{~b}$ compared to Figure 2a. It is interesting to find that the number concentrations of droplets with radius $(r)>\sim 3.75 \mu \mathrm{m}$ decrease when $\lambda$ increases from $\left\langle\sim 0.32 \mathrm{~km}^{-1}\right.$ to $>\sim 0.32 \mathrm{~km}^{-1}$, but the small droplets $(r<\sim 3.75 \mu \mathrm{m})$ increase. This inspires us to study the number concentration variations with $\lambda$ for different droplet size ranges in more details. Thus CDSDs are divided into three portions and the first portion has $r<3.75 \mu \mathrm{m}\left(N_{1}\right.$, the first two bins in CDSD). The other thirteen bins are divided into two approximately comparable parts $\left(N_{2}\right.$ and $\left.N_{3}\right)$, with $r$ between 3.75-12.75 $\mu \mathrm{m}$ (the middle six bins) and $12.75-23.25 \mu \mathrm{m}$ (the last seven bins), respectively (Figure $2 \mathrm{~b}$ ). The relationships between $N_{1}, N_{2}, N_{3}$ and $\lambda$ are shown in Figures 3a-3c, respectively.

Figure 3 shows that the correlations between $\lambda$ and the number concentrations $\left(N_{1}, N_{2}\right.$ and $\left.N_{3}\right)$ in the three $r$ ranges are all significantly negative as a whole and the correlation coefficients $(R)$ for them are $-0.32,-0.49$ and -0.57 , respectively. This confirms the conclusions in Figure $2 \mathrm{a}$ that the entrainment-mixing processes reduce droplet concentrations of all sizes simultaneously. But this is inconsistent with some previous observations [e.g., Lu et al., 2013] and simulations [e.g., Su et al., 1998] which found that CDSDs broaden toward small droplets and thus the number concentration of small droplets increases in the entrainment-mixing process. Now the question boils down to how the negative correlations between $N_{1}, N_{2}, N_{3}$ and $\lambda$ lead to the negative correlation between $\varepsilon$ and $\lambda$.

\subsection{Analysis based on $\sigma$ and $r_{\mathrm{m}}$}

Because $\varepsilon$ is the ratio of $\sigma$ to $r_{\mathrm{m}}$, so the effect of entrainment on $\varepsilon$ should be the balance of the effects of $\lambda$ on $\sigma$ and $r_{\mathrm{m}}$. Inspection of the relationships between $\sigma$ and $\lambda$ (Figure 4a) and between $r_{\mathrm{m}}$ and $\lambda$ (Figure 4b) for the 99 clouds reveals that $\sigma$ is negatively correlated with $\lambda(R=-0.50)$ and the $R$ of the correlation between $r_{\mathrm{m}}$ and $\lambda$ is -0.32 . However, as $\lambda$ increases, the decreasing rate of $\sigma$ (the slope of linear fittting is -9.32) is larger than that of $r_{\mathrm{m}}$ (the slope of linear fittting is -6.12). It is the unbalance of the decreasing rates that leads to the decrease of $\varepsilon$ with increasing $\lambda$. In addition, 
although the correlation between $r_{\mathrm{m}}$ and $\lambda$ is negative (Figure $4 \mathrm{~b}$ ), the negative correlation is mainly caused by the six clouds with $r_{\mathrm{m}}<\sim 4.90 \mu \mathrm{m}$ in the bottom right corner of Figure $4 \mathrm{~b}$. If the six clouds were excluded, $R$ for the correlation between $r_{\mathrm{m}}$ and $\lambda$ would be only -0.07 (93 clouds). The correlation coefficients for the relationships between $\sigma$ and $\lambda$, and between $\varepsilon$ and $\lambda$ in these 93 clouds would be -0.50 and -0.44 , respectively. This further confirms that the negative correlation between $\varepsilon$ and $\lambda$ is mainly due to the faster and stronger decrease of $\sigma$ compared to that of $r_{\mathrm{m}}$ as entrainment enhances if only based on mathematic relation.

As an absolute measure of spectral width of CDSD, $\sigma$ has also been extensively studied. The decrease of $\sigma$ with increasing $\lambda$ in Figure 4 a also seems in conflict with the traditional understanding that entrainment-mixing increases spectral width (Lasher-Trapp et al., 2005) or the results of approximately constant $\sigma$ in the process of enrainment-mixing [Lu et al., 2013; Pawlowska et al., 2006]. Recent studies on the correlation between $\sigma$ and entrainment were full of discrepancies. For example, Prabha et al., [2012] found that $\sigma$ reached its maximum in the less diluted regions of premonsoon clouds; but this trend was insignificant in monsoon clouds. The theoretical investigations of Pinsky et al., [2016] indicated that in the case of initially narrow CDSD and low relative humidity in environment, $\sigma$ increased and then decreased in the entrianment-mixing processes.

\subsection{Comparison of the roles of droplets with different sizes}

Detailed analyses are needed to combine the results in Sections 3.2 and 3.3 together and further explain how $\lambda$ influences CDSD, and then $\sigma, r_{\mathrm{m}}$ and $\varepsilon$ for these clouds. According to the influences of $\lambda$ on CDSD and cloud droplets of different sizes discussed in Section 3.2, the variations of $\sigma, r_{\mathrm{m}}$ and $\varepsilon$ with the number concentrations of the three sized droplet populations $\left(N_{1}, N_{2}\right.$ and $\left.N_{3}\right)$ can provide some clues (Figure 5).

The scatter plots in Figure 5 indicate that, the variation of $\sigma$ depends mainly on $N_{3}$ (Figures 5a-5c). The $R$ value of $\sigma$ vs. $N_{3}$ for these clouds in Figure 5c is 0.88 . The increased $\sigma$ with increasing large droplets was also found in Prabha et al., [2012]. Given the influences of $\lambda$ on CDSD in Figure 2a, the inhibition of $\lambda$ on $N_{3}$ causes a decrease in $\sigma$. As expected, $r_{\mathrm{m}}$ decreases with decreasing $N_{3}$, but increases with decreasing $N_{1}$ (Figures $5 \mathrm{~d}-5 \mathrm{f}$ ). Thøalance of the two 
opposite effects between $N_{1}$ and $N_{3}$ resulting from entrainment leads to the little variation of $r_{\mathrm{m}}$ with increasing $\lambda$ for the major 93 clouds among 99 clouds (Figure 4b). Based on the above analyses in Section 3.3, the effects of $\lambda$ on $\sigma$ and $r_{\mathrm{m}}$ jointly lead to the negative correlation between $\varepsilon$ and $\lambda$. Furthermore, in view of that entrainment-mixing necessarily reduce $N_{3}$, so the degree of evaporation of small droplets (in size bin of $N_{1}$ ) could be the real factor that regulates and controls the variation of $r_{\mathrm{m}}$ in the process of entrainment-mixing. When the evaporation is incomplete, CDSD broadens toward small size; but when the evaporation is complete, small droplets are removed and $r_{\mathrm{m}}$ tends to increase. Therefore, the incomplete and complete evaporation of small droplets (in size bin of $N_{1}$ ) could lead to the increase and decrease of $r_{\mathrm{m}}$, respectively and further different variation of $\varepsilon$.

It is also interesting to compare the roles of number concentrations of the droplets with different sizes on $\varepsilon$. Figure $5 \mathrm{~g}$ shows that $\varepsilon$ is positively correlated with $N_{1}(R=0.62)$. With the increase of $N_{1}, \sigma$ shows little variation but $r_{\mathrm{m}}$ significantly decreases, and this results in a positive correlation between $\varepsilon$ and $N_{1}$. In contrast to $N_{1}, \varepsilon$ shows little variation with $N_{2}$ and $N_{3}$ (Figures $5 \mathrm{~h}$ and 5 i). Although $\varepsilon$ is still positively correlated with $N_{3}$, the data points are quite scattered. In these clouds, the slope (0.95) of $\sigma$ vs. $N_{3}$ is relatively comparable with that (1.25) of $r_{\mathrm{m}}$ vs. $N_{3}$, which results in the insignificant correlation between $\varepsilon$ and $N_{3}$ in Figure 5i. Similar reasons are responsible for the marginal correlation between $\varepsilon$ and $N_{2}$ in these clouds. These results suggest that $N_{1}$ is more important than $N_{2}$ and $N_{3}$ in determining the influence of entrainment on $\varepsilon$. In addition, $N_{3}$ is a more important factor affecting $\sigma$ than $N_{1}$ and $N_{2}$.

\subsection{Further discussions}

Furthermore, the above analyses show that the clouds analyzed in this study exhibit decreasing trends of $N_{1}$ with increasing $\lambda$. But $\mathrm{Lu}$ et al., [2013] observed that the number concentration of small droplets in CDSD increases as entrainment enhances and $\varepsilon$ is positively correlated with $\lambda$. In fact for the relatively small cloud droplets in CDSD in this study, there are two inconsistent effects of entrainment on them because of different degree of evaporation of small droplets as mentioned above. On one hand, the increase of the number concentration of small droplets is accompanied with the size reduction of large droplets due to evaporation after 
entrainment-mixing, consequently leading to the increase of the concentration of small droplets (Figure 6a). Under this circumstance, the evaporation of small droplets is incomplete, $\sigma$ increases but $r_{\mathrm{m}}$ decreases. This is similar to the traditional understanding about the CDSD evolution in the process of entrainment-mixing and this corresponds to the positive correlation between $\varepsilon$ and $\lambda$. On the other hand, entrainment-induced dilution and evaporation can also reduce the sizes and number concentrations of small droplets. In this case, the evaporation of small droplets is complete, consequently leading to the decrease of small droplets (Figure 6b), and this corresponds to the negative correlation between $\varepsilon$ and $\lambda$ in this study. The mechanism is that because $\sigma$ mainly reduces with the decrease in of large droplets but shows little variation with small droplets (Figures 5a-5c), thus $\sigma$ significantly decreases when entrainment-mixing inhibits the concentration of large droplets. As shown in Figure 6b, all droplets with different sizes decrease as entrainment enhances, thus $r_{\mathrm{m}}$ has little change and consequently $\varepsilon$ decreases $\left(\varepsilon=\sigma r_{\mathrm{m}}\right)$. In a word, in the view of that large droplets necessarily decrease as entrainment enhances, the ultimate effects of entrainment on the number concentration of small droplets and the variation of $\varepsilon$ could mainly depend on the degree of evaporation of small cloud droplets. As mentioned above, $\varepsilon$ is crucial for precipitation. So the effect of entrainment on CDSD leads to the decrease of $\varepsilon$ and might reduce the probability of the coexistence of cloud droplets with different sizes, which goes against the collision-coalescence process of cloud droplets and the initiation of precipitation.

The decrease of $N_{1}$ with $\lambda$ also reveals that for these deep convective clouds, there existed some small cloud droplets in CDSD when the cloud parcels arrive at the observation levels. It is these already existed small droplets that make it possible for dilution or evaporation to decrease $N_{1}$. These small droplets might be related to secondary mixing events [Lu et al., 2014] or to reactivation of entrained aerosol particles [Su et al., 1998; Lasher-Trapp et al., 2005]. Because of the existence of small droplets, the CDSDs are wider than theoretically calculated CDSDs. But for narrow CDSDs, for example, in an adiabatic condition, the sizes of all droplets gather within a narrow $r$ range. Entrainment-mixing leads to the broadening of CDSD toward smaller sizes. This variation of CDSD in the entrainment-mixing processes is similar to the variation trend in Figure 6a, i.e., an increase of small droplets. So the traditional understanding for the CDSD evolution in the process of entrainment-mixing is mainly based on the theoretical adiabatic CDSD and this necessarily results in the positive correlation between $\varepsilon$ and $\lambda$ [Burnet and Brenguier. 2007]. 
Therefore, it can be speculated that the promoting effect of entrainment on precipitation is also mainly based on the initially narrow CDSD (shown in blue in Figure 6a), but not the CDSD in Figure $6 \mathrm{~b}$. In a word, the effect of entrainment-mixing processes on $\varepsilon$ is complicated, depending on the initial CDSD shapes and the competition of the aforementioned different effect of entrainment on CDSD.

In addition, it is also interesting to relate entrainment-mixing mechanisms, i.e., homogeneous and inhomogeneous mixing [Baker and Latham. 1979; Baker et al., 1980] to $\varepsilon$. Usually the relationship between volume-mean radius $\left(r_{\mathrm{v}}\right)$ and LWC can be used to judge the entrainment-mixing types. When mixing is homogeneous, all droplets evaporate at the same time with the dilution of the entrained air. So $r_{\mathrm{v}}$ reduces with the decreasing LWC. But for extreme inhomogeneous mixing, some droplets evaporate completely but the others are not influenced at all. Thus $r_{\mathrm{v}}$ does not change with the decreasing LWC. As analyzed in Guo et al., [2015], homogeneous mixing dominates in the clouds analyzed. However, Lu et al., [2013] found an increase of $\varepsilon$ with increasing $\lambda$ in the shallow cumuli wherein homogeneous mixing dominated. The theoretical study of Pinsky et al., [2016] indicated that the variation of $\varepsilon$ in the process of homogeneous mixing was uncertain and depended on the environmental relative humidity and the initial properties of CDSD. There is a need to consider $\varepsilon$ in investigation of entrainment-mixing processes [Yum et al., 2015], but it is unclear now how $\varepsilon$ changes in homogeneous entrainment-mixing processes. This topic is still in its infancy and deserves further studies in the future to examine.

\section{Conclusions}

Entrainment can significantly influence the microphysical characteristics of cloud droplet size distribution (CDSD). Relative dispersion $(\varepsilon)$ is a crucial microphysical parameter affecting cloud properties. So the influence of entrainment on $\varepsilon$ of CDSD in the 99 identified growing deep convective clouds observed at the $\sim 3000 \mathrm{~m}$ height from the TOGA-COARE field campaign is examined in this study.

The results indicate that in these clouds, the correlation between $\varepsilon$ and $\lambda$ is negative, i.e., entrainment suppresses the $\varepsilon$ of CDSD. This is opposite to the traditional understanding that 
entrainment broadens CDSD toward smaller droplets, i.e., $\varepsilon$ increases in the entrainment-mixing processes. In order to study the cloud droplet number concentration variations with $\lambda$ for different droplet size ranges in more detail, CDSDs are divided into three portions with droplet radius < $3.75 \mu \mathrm{m}\left(N_{1}\right)$, radius in the range of 3.75-12.75 $\mu \mathrm{m}\left(N_{2}\right)$ and $12.75-23.25 \mu \mathrm{m}\left(N_{3}\right)$, respectively. The further analyses indicate that the number concentrations of all cloud droplets with different sizes decrease simultaneously as $\lambda$ increases. This is also inconsistent to some previous observations [e.g., Lu et al., 2013] and simulations [e.g., Su et al., 1998] which found that CDSD broaden toward small droplets and thus the number concentration of small droplets increase in the entrainment-mixing process.

In these 99 clouds, the variation of $\sigma$ depends mainly on $N_{3}$, while $r_{\mathrm{m}}$ reduces with the decrease of $N_{3}$, but increases with decreasing $N_{1}$, so the influence of entrainment on CDSD causes a decrease in $\sigma$ but little variation in $r_{\mathrm{m}}$; thus $\varepsilon$ generally reduces as $\lambda$ increases for these clouds. Furthermore, the comparison of the roles of droplets with different sizes reveals that $N_{1}$ is more important than $N_{2}$ and $N_{3}$ in determining the influence of entrainment on $\varepsilon$. But $N_{3}$ is a more important factor affecting $\sigma$ than $N_{1}$ and $N_{2}$. In a word, the effect of entrainment on CDSD leads to the decrease of $\varepsilon$ and might go against the collision-coalescence process of cloud droplets and precipitation. Finally, a conceptual model of CDSD evolutions during entrainment-mixing processes is developed to show the scenarios where the relationship between $\varepsilon$ and $\lambda$ is positive or negative; the conceptual model further indicates that the small droplet and the degree of evaporation of small droplets are key factors shifting the positive or negative relationship. It is speculated that the promoting effect of entrainment on precipitation is mainly based on initially narrow CDSD.

\section{Acknowledgements}

This research was supported by the National Key Research and Development Program of China (2017YFA0604000), the National Natural Science Foundation of China (NSFC) (91537108), China Meteorological Administration Special Public Welfare Research Fund (GYHY201406001, GYHY201406007), the Natural Science Foundation of Jiangsu Province, China (BK20160041), the U.S. Department oEnergy's BER Atmospheric SystemResearch (ASR) 
High-level Talents Training Project in Jiangsu (BRA2016424), NSFC(41305120). All the data are from TOGA-COARE (Tropical Ocean Global Atmosphere Coupled Ocean Atmosphere Response Experiment) field campaign (http://data.eol.ucar.edu/codiac/dss/id=2.1500), including microphysical, dynamical and thermodynamic properties.

\section{References}

Baker, M. B., J., Latham (1979), The evolution of droplet spectra and the rate of production of embryonic raindrops in small cumulus clouds. J. Atmos. Sci. 36(8), 1612-1615, doi:10.1175/1520-0469(1979)036<1612:TEODSA>2.0.CO;2.

Beals, M. J., J. P., Fugal, R. A., Shaw, J., Lu, S. M., Spuler, and J. L., Stith (2015), Holographic measurements of inhomogeneous cloud mixing at the centimeter scale. Science. 350, 87-90, doi: 10.1126/science.aab0751.

Burnet, F., and J. L., Brenguier (2007), Observational study of the entrainment-mixing process in warm convective clouds. J. Atmos. Sci. 64 (6), 1995-2011, doi:10.1175/JAS3928.1.

Böing, S. J., H. J. J., Jonker, W. A., Nawara, and A. P., Siebesma (2014), On the deceiving aspects of mixing diagrams of deep cumulus convection. J. Atmos. Sci. 71(1), 56-68, doi: dx.doi.org/10.1175/JAS-D-13-0127.1.

Chen, J., Y., Liu, M., Zhang, and Y., Peng (2016), New understanding and quantification of the regime dependence of aerosol-cloud interaction for studying aerosol indirect effects. Geophys. Res. Lett. 43, 1780-1787, doi: 10.1002/2016GL067683.

Chen, J., X., Wu, Y., Yin, Q., Huang, and H., Xiao (2017), Characteristics of Cloud Systems over the Tibetan Plateau and East China during Boreal Summer, J. Climate. 30(9), 3117-3137, doi: doi.org/10.1175/JCLI-D-16-0169.1.

Chen, Q., I., Koren, O., Altaratz, R. H., Heiblum, G., Dagan, and L., Pinto (2017), How do changes in warm-phase microphysics affect deep convective clouds?, Atmos. Chem. Phys. 17(15), doi: 9585-9598, 10.5194/acp-17-9585-2017.

Del Genio, A. D., and J., Wu (2010), The role of entrainment in the diurnal cycle of continental convection, J. Climate. 23(10), 2722-2738, doi: 10.1175/2009JCLI3340.1.

Gerber, H. E., G. M., Frick, J. B., Jensen, and J. G., Hudson (2008), Entrainment, mixing, and microphysics in trade-wind cumulus. J. Meterol. Soc. Jpn. 86, 87-106.

Grabowski, W.W., and H., Pawlowska (1993), Entrainment and mixing in clouds, The Paluch mixing diagram revisited. J. Appl. Meterol. 32 (11), 1767-1773, doi: http://dx.doi.org/10.1175/1520-0450(1993)032<1767:EAMICT>2.0.CO;2.

Guo, X., C., Lu, T., Zhao, G. J., Zhang, and Y., Liu (2015), An observational study of entrainment rate in deep convection. Atmosphere. 6, 1362-1376, doi:10.3390/atmos6091362. 
Khairoutdinov, M., and D., Randall (2006), High-resolution simulation of shallow to deep convection transition over land. J. Atmos. Sci. 63, 3421-3436, doi: dx.doi.org/10.1175/JAS3810.1.

Kumar, B., J., Schumacher, and R. A., Shaw (2014), Lagrangian mixing dynamics at the cloudy-clear air interface, J. Atmos. Sci. 71(7), 2564-2580, doi: 10.1175/JAS-D-13-0294.1.

Lasher-Trapp, S.G., W. A., Cooper, and A. M., Blyth (2015), Broadening of droplet size distributions from entrainment and mixing in a cumulus cloud. Quart. J. Roy. Meteor. Soc. 131, 195-220, doi: 10.1256/qj.03.199.

Liu, Y., and J., Hallett (1998), On size distributions of cloud droplets growing by condensation: A new conceptual model. J. Atmos. Sci. 55, 527-536, doi: http://dx.doi.org/10.1175/1520-0469(1998)055<0527:OSDOCD>2.0.CO;2.

Liu, Y., and P. H., Daum (2000), Spectral dispersion of cloud droplet size distributions and the parameterization of cloud droplet effective radius. Geophys. Res. Lett. 27, 1903-1906.

Liu, Y., and P. H., Daum (2002), Indirect warming effect from dispersion forcing. Nature. 419, 580-581.

Liu, Y., P. H., Daum, and R., McGraw (2005), Size truncation effect, threshold behavior, and a new type of autoconversion parameterization. Geophys. Res. Lett. 32, L11811, doi:10.1029/2005GL022636.

Liu, Y., P. H., Daum, R., McGraw, and M., Miller (2006), Generalized threshold function accounting for effect of relative dispersion on threshold behavior of autoconversion process. Geophys. Res. Lett. 33, L11804, doi:10.1029/2005GL025500.

Liu, Y., P. H., Daum, H., Guo, and Y., Peng (2008), Dispersion bias, dispersion effect, and the aerosol-cloud conundrum. Environ. Res. Lett. 3(4), 045021, doi:10.1088/1748-9326/3/4/045021.

Liu, Y., P. H., Daum, and C., Lu (2014), Comment on "Cloud droplet spectral width relationship to CCN spectra and vertical velocity" by Hudson et al., J. Geophys. Res. Atmos. 119, 1874-1877, doi:10.1029/2012JD017546.

Lin, W., Y., Liu, A. M., Vogelmann, A. Fridlind, S. Endo, H. Song, S. Feng, T. Toto, Z. Li and M. Zhang (2015). "RACORO continental boundary layer cloud investigations: 3. Separation of parameterization biases single-column model CAM5 simulations of shallow cumulus." J. Geophys. Res. Atmos. 120(12): 6015-6033. doi: 10.1002/2014JD022525.

Lu, C., Y., Liu, S., Niu, and A. M., Vogelmannn (2012a), Observed impacts of vertical velocity on cloud microphysics and implications for aerosol indirect effects. Geophys. Res. Lett. 39, L20808, doi:10.1029/2012GL053599.

Lu, C., Y., Liu, S., Niu, and A. M., Vogelmannn (2012b), Lateral entrainment rate in shallow cumuli, Dependence on dry air sources and probability density functions. Geophys. Res. Lett. 39, L20812, doi:10.1029/2012GL053646. 
Lu, C., Y., Liu, S., Niu, and A. M., Vogelmannn (2013), Empirical relationship between entrainment rate and microphysics in cumulus clouds. Geophys. Res. Lett. 40, 2333-2338, doi:10.1002/grl.50445.

Lu, C., Y., Liu, and S., Niu (2014), Entrainment-mixing parameterization in shallow cumuli and effects of secondary mixing events. Chin. Sci. Bull. 59(9), 896-903, doi: 10.1007/s11434-013-0097-1.

Lu, C., Y., Liu, G., Zhang, X., Wu, S., Endo, L., Cao, Y., Li, X., Guo (2016), Improving Parameterization of Entrainment Rate for Shallow Convection with Aircraft Measurements and Large-Eddy Simulation. J. Atmos. Sci., 73: 761-773, doi: http://dx.doi.org/10.1175/JAS-D-15-0050.1.

Luo, Z., Y., Liu and G. L., Stephens (2010), Use of A-Train data to estimate convective buoyancy and entrainment rate. Geophys. Res. Lett. 37, 384-397, doi: 10.1029/2010GL042904.

Ma, J., Y., Chen, W., Wang, P., Yan, H., Liu, S., Yang, and J., Lelieveld (2010), Strong air pollution causes widespread haze-clouds over China. J. Geophys. Res. Atmos. 115, D18204, doi: 10.1029/2009JD013065.

Martin, G. M., D. W., Johnson, and A., Spice (1994), The measurement and parameterization of effective radius of droplets in the warm stratocumulus clouds. J. Atmos. Sci. 51, 1823-42, doi: http://dx.doi.org/10.1175/1520-0469(1994)051<1823:TMAPOE>2.0.CO;2.

Martin, S. S., and A. O., Paul (2013), Influence of entrainment on the thermal stratification in simulations of radiative-convective equilibrium. Geophys. Res. Lett. 40, 5223-5227, doi:10.1002/grl.50796.

McFarquhar, G. M., and A. J., Heymsfield (2001), Parameterizations of INDOEX microphysical measurements and calculations of cloud susceptibility, Applications for climate studies. J. Geophys. Res. 106, 28, doi: 10.1029/2000JD900777.

Neggers R.A.J., A.P., Siebesma, H.J.J., Jonker (2002), A multiparcel model for shallow cumulus $\begin{array}{lllll}\text { convection. } & \mathrm{J} & \text { Atmos }\end{array}$ doi: http://dx.doi.org/10.1175/1520-0469(2002)059<1655:AMMFSC>2.0.CO;2.

Norgren, M.S., J. D., Small, H. H., Jonsson, and P. Y. ,Chuang (2016), Observational estimates of detrainment and entrainment in non-precipitating shallow cumulus. Atmos. Chem. Phys. 16(1), 21-33, doi:10.5194/acpd-14-21785-2014.

Pawlowska, H., W. W., Grabowski, and J. L., Brenguier (2006), Observations of the width of cloud droplet spectra in stratocumulus, Geophys. Res. Lett. 33, L19810, doi:10.1029/2006GL026841.

Peng, Y., and U., Lohmann (2003), Sensitivity study of the spectral dispersion of the cloud droplet size distribution on the indirect aerosol effect. Geophys. Res. Lett. 30(10), doi:10.1029/2003GL017192. 
Pinsky, M., A., Khain, A., Korolev, and L., Magaritz-Ronen (2016), Theoretical investigation of mixing in warm clouds-Part 2, Homogeneous mixing. Atmos. Chem. Phys. 16, 9255-9272, doi:10.5194/acp-16-9255-2016.

Prabha, T. V., S., Patade, G., Pandithurai, A., Khain, D., Axisa, P., Pradeep-Kumar, and R. S., Maheshkumar (2012), Spectral width of premonsoon and monsoon clouds over Indo-Gangetic valley. J. Geophys. Res. Atmos. 117, D20205, doi:10.1029/2011JD016837.

Seifert, A., and K. D., Beheng (2001), A double-moment parameterization for simulating autoconversion, accretion and selfcollection. Atmos. Res. 59, 265-281.

Shi, X., and X., Liu (2016), Effect of cloud-scale vertical velocity on the contribution of homogeneous nucleation to cirrus formation and radiative forcing, Geophys. Res. Lett. 43(12), 6588-6595, doi: 10.1002/2016GL069531.

Su, C. W., S. K., Krueger, P. A., McMurtry, and P. H., Austin (1998), Linear eddy modeling of droplet spectral evolution during entrainment and mixing in cumulus clouds, Atmos. Res., 1998, 47, 41-58.

Tas, E., A., Teller, O., Altaratz, D., Axisa, R., Bruintjes, Z., Levin, and I., Koren (2015), The relative dispersion of cloud droplets, its robustness with respect to key cloud properties. Atmos. Chem. Phys. 15(4), 2009-2017, doi:10.5194/acpd-14-11153-2014.

Tölle, M. H., and S. K., Krueger (2014), Effects of entrainment and mixing on droplet size distributions in warm cumulus clouds. J Adv. Model. Earth Syst. 6(2), 281-299, doi: 10.1002/2012MS000209.

Webster, P. J., and R., Lukas (1992), TOGA COARE: The coupled ocean-atmosphere response experiment. Bulletin of the American Meteorological Societ. 73(9), 1377-1416, doi: http://dx.doi.org/10.1175/1520-0477(1992)073<1377:TCTCOR>2.0.CO;2.

Wood, R., S., Irons, and P. R., Jonas (2002), How important is the spectral ripening effect in stratiform boundary layer clouds? Studies using simple trajectory analysis. J. Atmos. Sci. 59 (18),2681-2693,doi: http://dx.doi.org/10.1175/1520-0469(2002)059<2681:HIITSR>2.0.CO;2

Yang, F., R., Shaw, and H., Xue (2016), Conditions for super-adiabatic droplet growth after entrainment mixing, Atmos. Chem. Phys., 16, 9421-9433, doi: 10.5194/acp-2016-94.

Xie, X., X., Liu, Y., Peng, Y., Wang, Z. Yue and X., Li (2013), Numerical simulation of clouds and precipitation depending on different relationships between aerosol and cloud droplet spectral dispersion. Tellus B. 65, doi: http://dx.doi.org/10.3402/tellusb.v65i0.19054.

$\mathrm{Xu}, \mathrm{X}$., and H., Xue (2015), Impacts of free-tropospheric temperature and humidity on nocturnal nonprecipitating marine stratocumulus. J. Atmos. Sci. 72(8), 2853-2864, doi: http://dx.doi.org/10.1175/JAS-D-14-0387.1.

Yum, S. S., and J. G., Hudson (2005), Adiabatic predictions and observations of cloud droplet spectral broadness, Atmos. Res. 73, 203-223, doi:10.1016/j.atmosres.2004.10.006.

Yum, S. S., J., Wang, Y., Liu, G., Senum, S., Springston, R., McGraw, and J.M., Yeom (2015), Cloud microphysical relationships and their implication on entrainment and mixing 
mechanism for the stratocumulus clouds measured during the VOCALS project. J. Geophys. Res. Atmos. 120(10), 5047-5069, doi: 10.1002/2014JD022802.

Zhang, F., K., Wu, J., Li, Q., Yang, J. Q., Zhao, and J., Li (2016), Analytical Infrared Delta-Four-Stream Adding Method from Invariance Principle. J. Atmos. Sci. 73(10), 4171-4188, doi: doi.org/10.1175/JAS-D-15-0317.1.

Zhang, F., Y. N., Shi, J., Li, K., Wu, and H., Iwabuchi (2017a), Variational Iteration Method for Infrared Radiative Transfer in a Scattering Medium. J. Atmos. Sci. 74(2), 419-430, doi: doi.org/10.1175/JAS-D-16-0172.1.

Zhang, F., K., Wu, P., Liu, X., Jing, and J., Li (2017b), Accounting for Gaussian quadrature in four-stream radiative transfer algorithms, Journal of Quantitative Spectroscopy \& Radiative Transfer, 192, 1-13, doi: doi.org/10.1016/j.jqsrt.2017.01.040.Zhang, G. J., X., Wu, X., Zeng, and T., Mitovski (2015), Estimation of convective entrainment properties from a cloud-resolving model simulation during TWP-ICE. Clim. Dyn. 1-16, doi: 10.1007/s00382-015-2957-7.

Zhao, C., X., Tie, G., Brasseur, K. J., Noone, T., Nakajima, Q., Zhao, Y., Ishizaka (2006). Aircraft measurements of cloud droplet spectral dispersion and implications for indirect aerosol radiative forcing. Geophys. Res. 33, L16809, doi:10.1029/2006GL026653. 


\section{Caption Lists}

Figure 1. Scatter plot of entrainment rate $\lambda$ l vs. relative deviation () of CDSD in the total 99 clouds. Legend provides the correlation coefficient $(R)$ and $p$ values for correlation.

Figure 2. Average CDSDs correspond to different entrainment rate $(\lambda)$ for the total 99 clouds when CDSDs are averaged in (a) three bins and (b) twelve bins. The abscissas and ordinates represent cloud droplet radius $(r)$ and $\mathrm{d} N / \mathrm{d} r$, respectively The color bars represent $\lambda$. These CDSDs are divided into three portions $\left(N_{1}, N_{2}\right.$ and $N_{3}$, based on droplet radius $<3.75 \mu \mathrm{m}\left(N_{1}\right)$, radius in the range of 3.75-12.75 $\mu \mathrm{m}\left(N_{2}\right)$ and $12.75-23.25 \mu \mathrm{m}\left(N_{3}\right)$, respectively) by two dashed vertical lines in (b). See text for more details.

Figure 3. Scatter plots of entrainment rate $(\lambda) v s$. number concentration of cloud droplets with radius (a) $<3.75 \mu \mathrm{m}\left(N_{1}\right)$, in the range of (b) $3.75-12.75 \mu \mathrm{m}\left(N_{2}\right)$ and (c) $12.75-23.25 \mu \mathrm{m}\left(N_{3}\right)$, respectively. Each legend also provides the correlation coefficient $(R)$ and $p$ values for each correlation.

Figure 4. Scatter plots of entrainment rate $\chi$ ) vs. (a) standard deviation $(\sigma)$ and (b) mean radius $\left(r_{\mathrm{m}}\right)$ of CDSD in the total 99 clouds. Each legend provides the correlation coefficienR)(and $p$ values for each correlation.

Figure 5. Scatter plots of standard deviation $(\sigma) v s$. number concentration of cloud droplets with radius (a) $<3.75 \mu \mathrm{m}\left(N_{1}\right)$, in the ranges of (b) 3.75-12.75 $\mu \mathrm{m}\left(N_{2}\right)$ and (c) $12.75-23.25 \mu \mathrm{m}\left(N_{3}\right)$, respectively for the total 99 clouds. (d)-(f) and (g)-(i) are the same as (a)-(c) but for mean radius $\left(r_{\mathrm{m}}\right)$ and relative dispersion $(\varepsilon) v s$. number concentration, respectively Each legend also provides the correlation coefficient $(R)$ and $p$ values for each correlation.

Figure 6. Conceptual evolutions of CDSDs (from the blue to the red CDSD) after entrainment-mixing processes for (a) initially narrow CDSD, and (b) the 99 clouds analyzed in this study. 


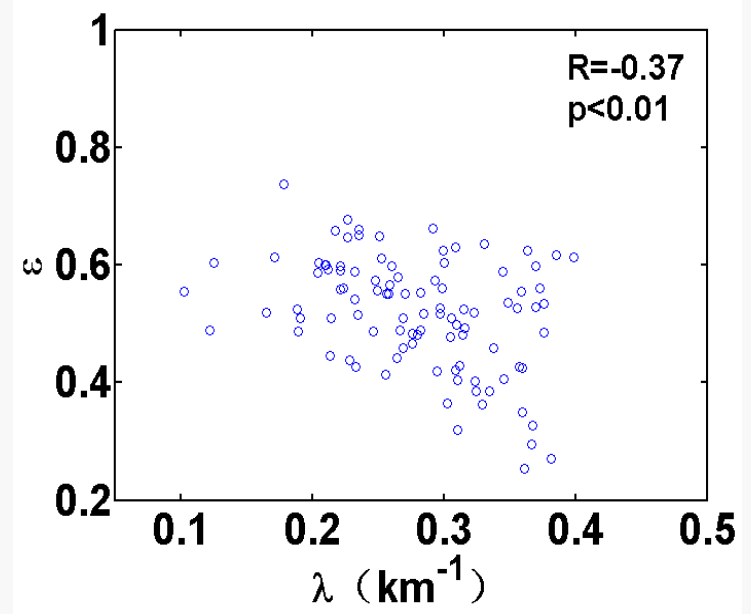

Figure 1. Scatter plot of entrainment rate $(\lambda) v s$. relative deviation $(\varepsilon)$ of CDSD in the total 99 clouds. Legend provides the correlation coefficient $(R)$ and $p$ values for correlation. 

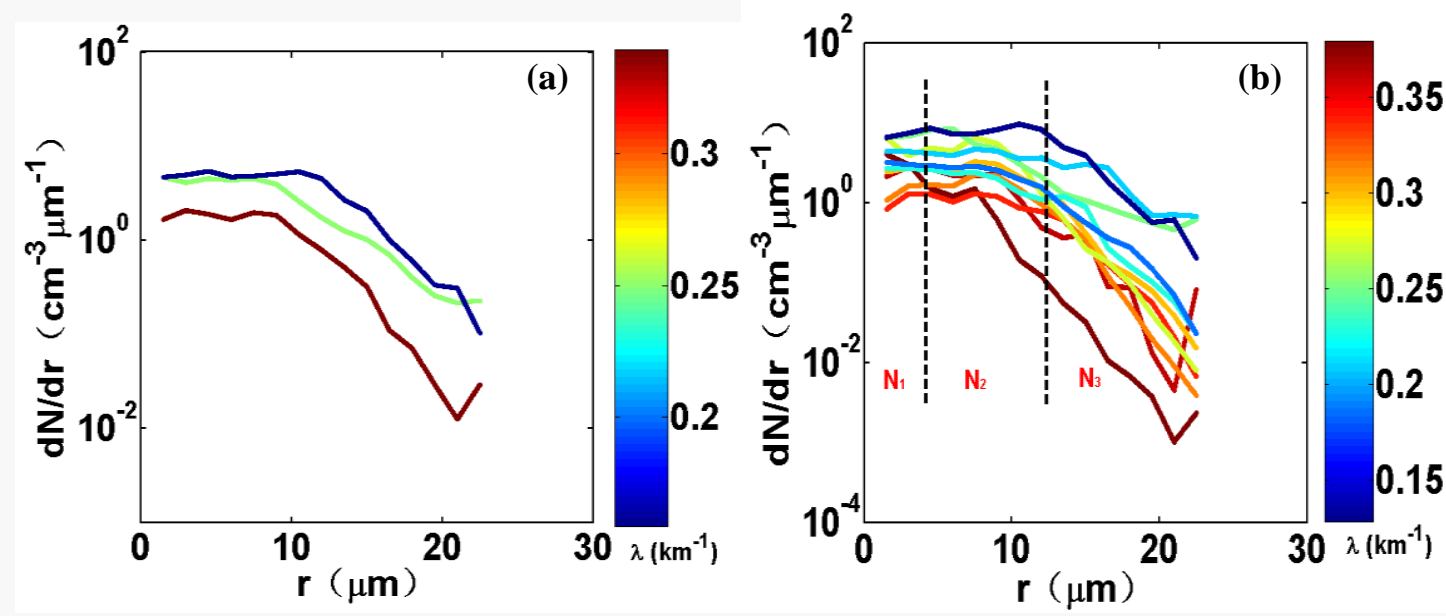

Figure 2. Average CDSDs correspond to different entrainment rate $(\lambda)$ for the total 99 clouds when CDSDs are averaged in (a) three bins and (b) twelve bins. The abscissas and ordinates represent cloud droplet radius $(r)$ and $\mathrm{d} N / \mathrm{d} r$, respectively. The color bars represent $\lambda$. These CDSDs are divided into three portions $\left(N_{1}, N_{2}\right.$ and $N_{3}$, based on droplet radius $<3.75 \mu \mathrm{m}\left(N_{1}\right)$, radius in the range of $3.75-12.75 \mu \mathrm{m}\left(N_{2}\right)$ and $12.75-23.25 \mu \mathrm{m}\left(N_{3}\right)$, respectively) by two dashed vertical lines in (b). See text for more details. 

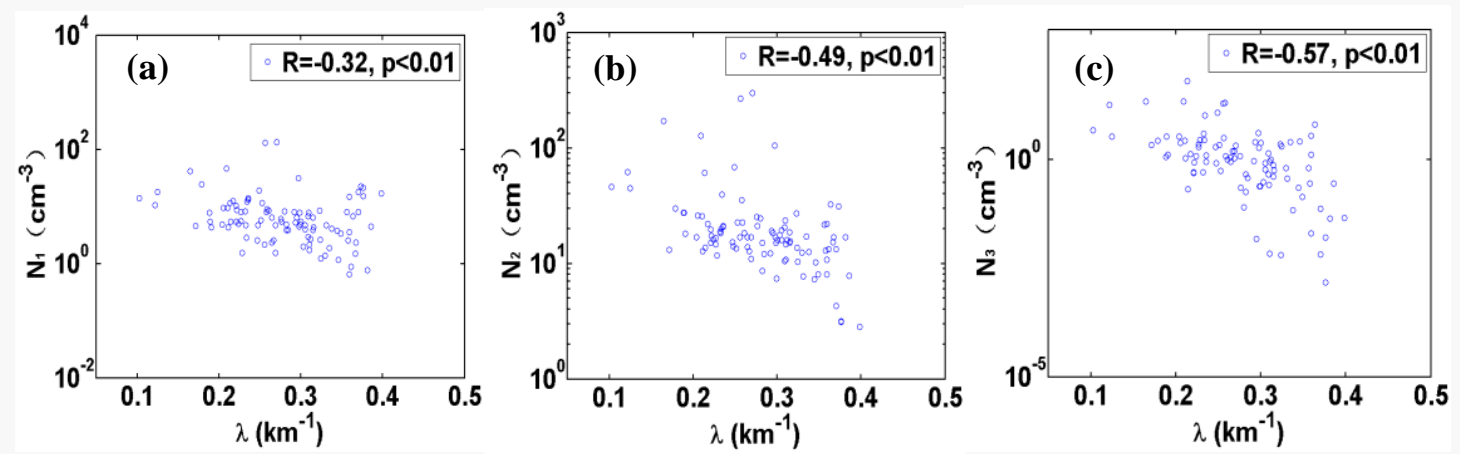

Figure 3. Scatter plots of entrainment rate $(\lambda) v s$. number concentration of cloud droplets with radius (a) $<3.75 \mu \mathrm{m}\left(N_{1}\right)$, in the range of (b) 3.75-12.75 $\mu \mathrm{m}\left(N_{2}\right)$ and (c) $12.75-23.25 \mu \mathrm{m}\left(N_{3}\right)$, respectively. Each legend also provides the correlation coefficient $(R)$ and $p$ values for each correlation. 

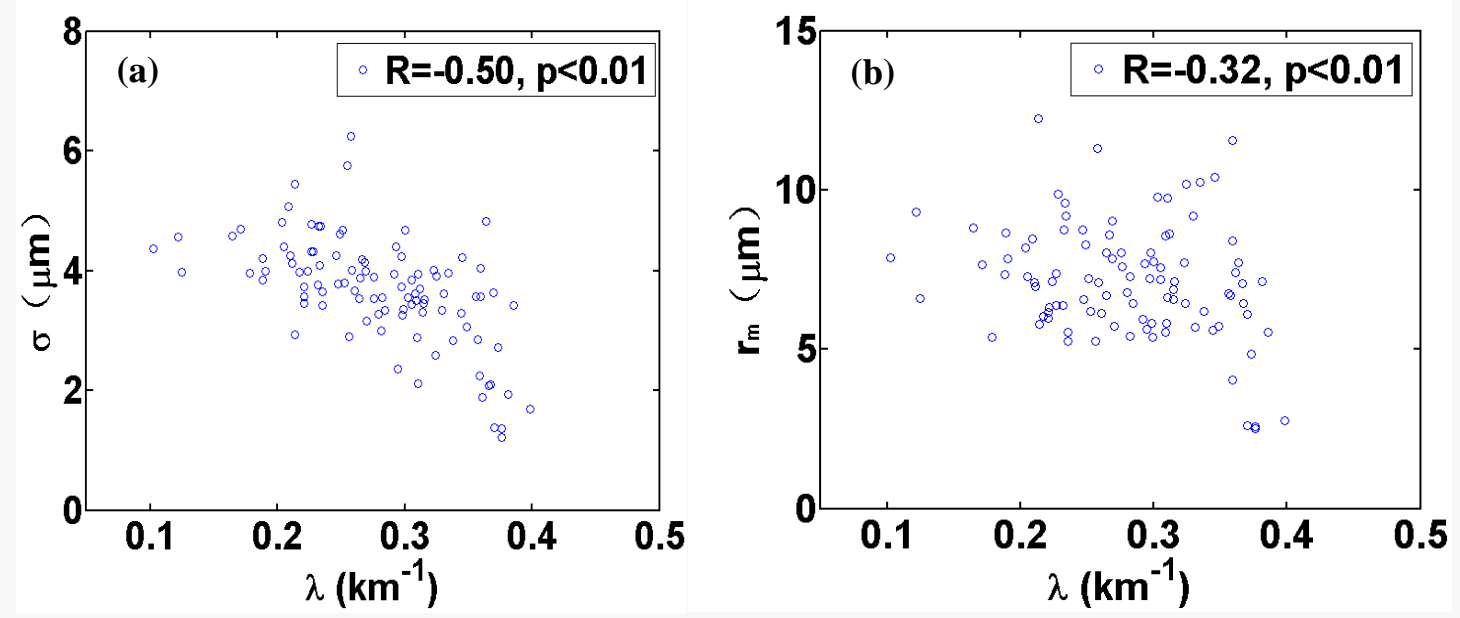

Figure 4. Scatter plots of entrainment rate $(\lambda)$ vs. (a) standard deviation $(\sigma)$ and (b) mean radius $\left(r_{\mathrm{m}}\right)$ of CDSD in the total 99 clouds. Each legend provides the correlation coefficient $(R)$ and $p$ values for each correlation. 

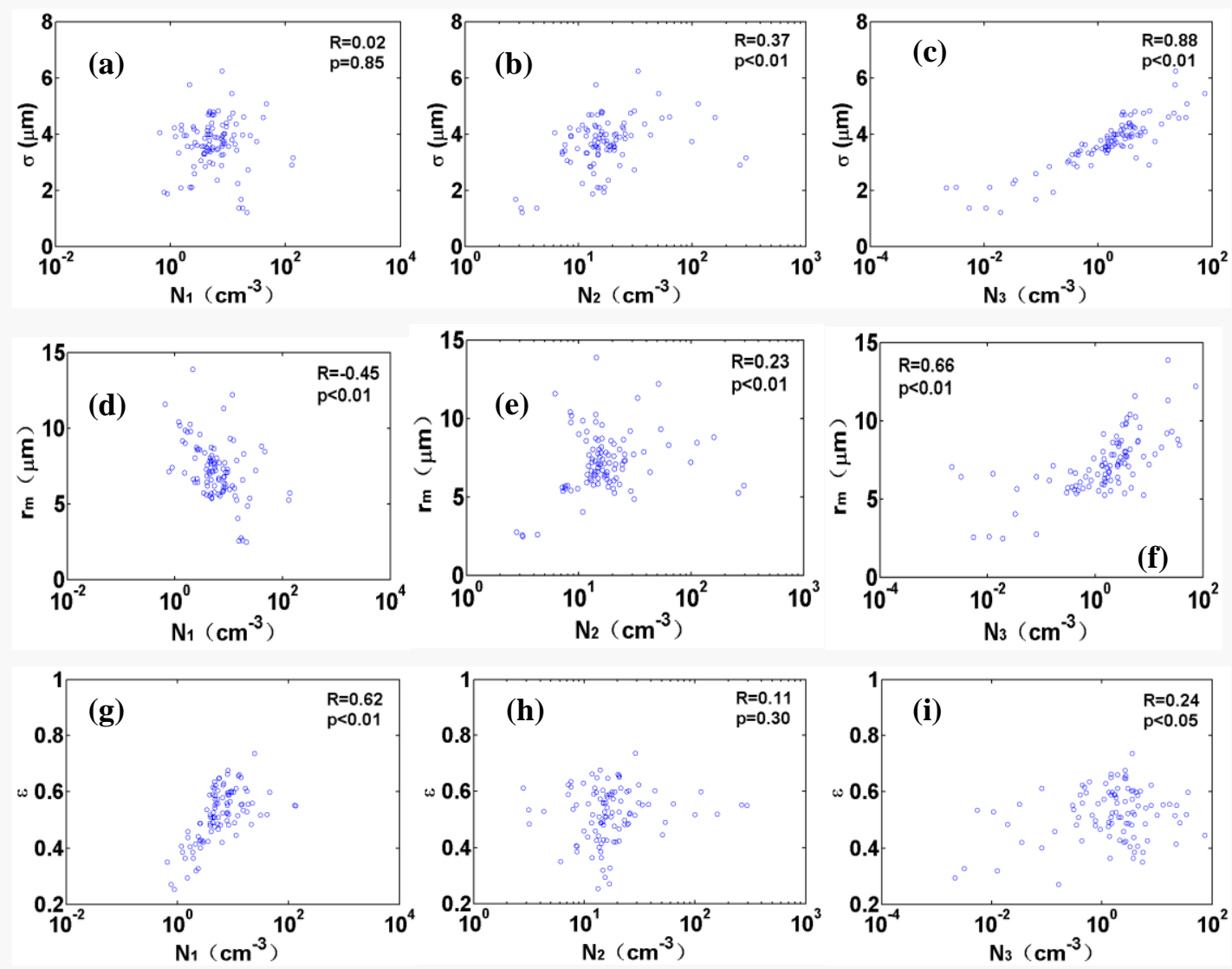

Figure 5. Scatter plots of standard deviation $(\sigma) v s$. number concentration of cloud droplets with radius (a) $<3.75 \mu \mathrm{m}\left(N_{1}\right)$, in the ranges of (b) $3.75-12.75 \mu \mathrm{m}\left(N_{2}\right)$ and (c) $12.75-23.25 \mu \mathrm{m}\left(N_{3}\right)$, respectively for the total 99 clouds. (d)-(f) and (g)-(i) are the same as (a)-(c) but for mean radius $\left(r_{\mathrm{m}}\right)$ and relative dispersion $(\varepsilon) v s$. number concentration, respectively. Each legend also provides the correlation coefficient $(R)$ and $p$ values for each correlation. 

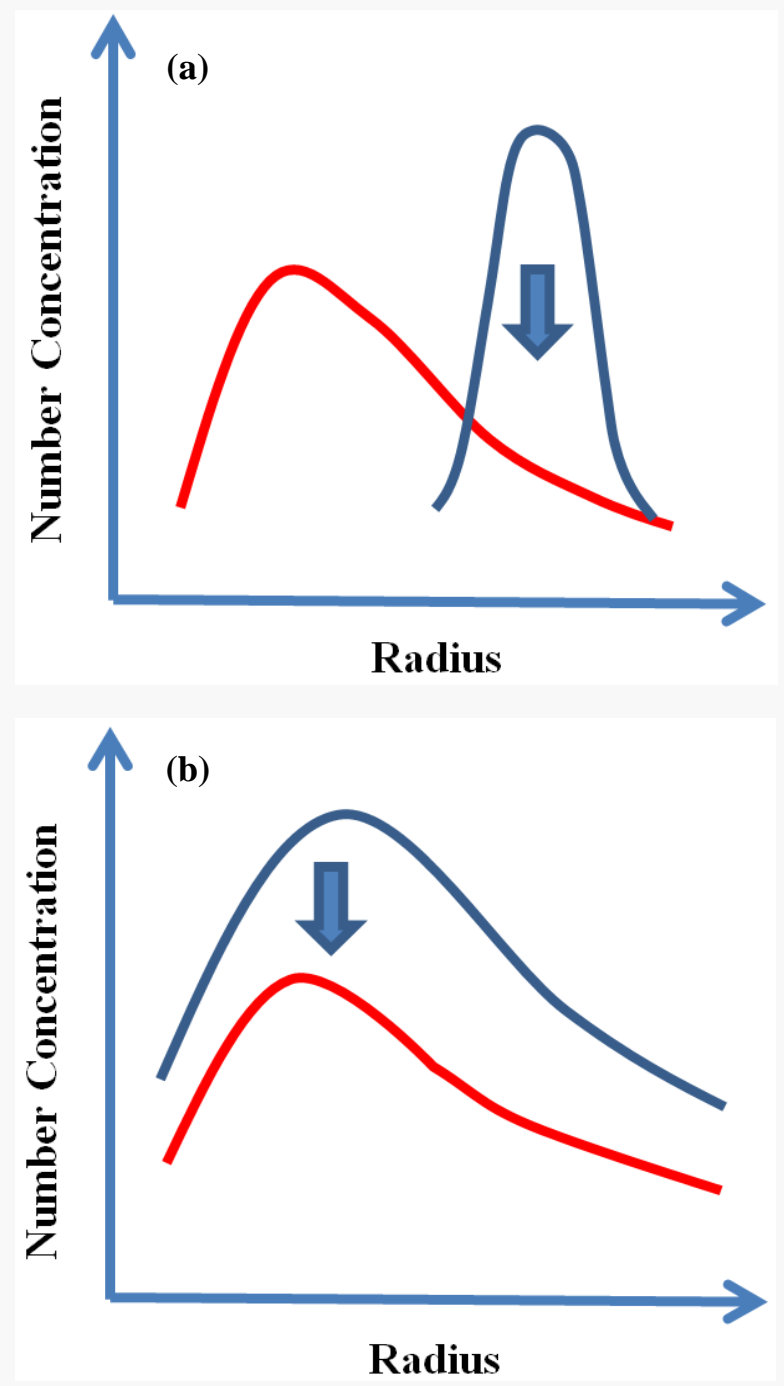

Figure 6. Conceptual evolutions of CDSDs (from the blue to the red CDSD) after entrainment-mixing processes for (a) initially narrow CDSD, and (b) the 99 clouds analyzed in this study. 
This study investigates the influence of entrainment rate on relative dispersion of cloud droplet size distribution in 99 clouds. Tothe author' knodedge, this is the first obsevatinal study on the relationship between relative dispersion and entrainment rate in deep convective clouds. It is found that relative dispersion is negatively correlated with entrainment rate, and this is opposite to the traditional understanding that entrainment-mixing broadens cloud droplet size distribution. Herein we examine the mechanisms for the negative correlation between relative dispersion and entrainment rate. A conceptual model of cloud droplet size distribution evolutions during entrainment-mixing processes is developed to illustrate the possible scenarios entailing different relationships between relative dispersion and entrainment rate. The correlation between entrainment-mixing mechanism and relative dispersion is also discussed in paper. 